\section{The implications of target saturation for the use of drug-target residence time}

\author{
Wilhelmus E. A. de Witte, Meindert Danhof, Piet H. van der Graaf and \\ Elizabeth C. M. de Lange
}

The interaction between a drug and its biological target is a key step in the causal chain between drug dosing and drug effect in the human body. The strength of this interaction may be represented by the drug-target dissociation constant $\left(K_{\mathrm{d}}\right)$, which describes the drug concentration that results in 50\% target occupancy (that is, the percentage of target molecules that are bound to a drug molecule) at equilibrium. However, the value of $K_{d}$ does not provide information on the rate at which target binding equilibrium is reached after a change in the drug concentration. The kinetics of target binding are most simply described by two rate constants: the second-order association rate constant $k_{\text {on }}$ and the first-order dissociation rate constant $k_{\text {off }}$ (FIG. 1a).

Drug-target residence time, defined as $1 / k_{\text {off }}$ has received increasing attention in drug discovery following the publication of an article by Copeland and colleagues in 2006 that discussed the beneficial effect of a long dissociative half-life of a drug-target complex (defined as $\ln [2] / k_{\text {off }}$ ) on (selective) prolongation of target occupancy and thus of pharmacological effects ${ }^{1}$. Since the publication of this paper, we and others have highlighted some limitations of the simple drug-target residence time model and the interpretation of its results; for examples, see REFS ${ }^{2-5}$. The aim of this article, which is based on previous research from our group ${ }^{4}$, is to illustrate the impact of the role of target saturation (that is, target occupancy close to $100 \%$ ) on the prolongation of target occupancy and to show that lack of consideration of this role may contribute to inaccurate conclusions about the influence of drug-target binding kinetics on the duration of target occupancy. In particular, a value of $k_{\text {off }}$ that is lower than the pharmacokinetic elimination rate constant $\left(k_{\mathrm{el}}\right)$ may not be the key determinant of the duration of target occupancy as the target becomes closer to being saturated. We also discuss examples that help illustrate how to take into account the role of target saturation in decisions about whether or not to select drug candidates with low $k_{\text {off }}$ values (that is, long drug-target residence times).

\section{Importance of target saturation}

When different compounds for which a reduction in $k_{\text {off }}$ is accompanied by an increased affinity are compared, increased in vivo duration of target occupancy is expected based on the increased affinity alone, if the tested concentrations are similar and lead to an initial target occupancy close to $100 \%$. As a consequence, the increased duration of drug action that is observed in such a comparison cannot be attributed to the $k_{\text {off }}$ but is purely dependent on the $K_{\mathrm{d}}$, the pharmacokinetics and the administered dose. An example of such a comparison is given in FIG. $1 \mathrm{~b}$, where we took the $k_{\text {off }}, k_{\text {on }}$ and elimination half-life values of the HIV protease inhibitors amprenavir, lopinavir and atazanavir from REF. ${ }^{5}$ and simulated their target occupancy. The compounds with the lowest $k_{\text {off }}$ values, lopinavir and atazanavir, also had increased affinities and showed an increased duration of target occupancy. As can be clearly seen in FIG. 1 b, the increased duration of target occupancy is only the consequence of a rightward shift in the occupancy-time curve and thus only the consequence of the $K_{\mathrm{d}}$. To demonstrate this, we overlaid the simulations of lopinavir and atazanavir with simulations of hypothetical compounds that have the same $K_{\mathrm{d}}$ values as lopinavir and atazanavir, but the $k_{\text {off }}$ value of amprenavir.

An example where a decrease in $k_{\text {off }}$ does lead to an additional increase in the duration of target occupancy compared with the affinity-driven increase in target occupancy is given in FIG. 1c. In this simulation, we took the $k_{\text {off }} k_{\text {on }}$ and elimination half-life values of ipratropium, aclidinium and PF-3635659 from REF. ${ }^{5}$ and REF. ${ }^{6}$ and simulated their target occupancy at the muscarinic $\mathrm{M}_{3}$ receptor. The compounds with the lowest $k_{\text {off }}$ values, aclidinium and PF-3635659, also had increased affinities and showed an increased duration of target occupancy. However, the increased duration of target occupancy in FIG. 1c is not only the consequence of a rightward shift in the occupancy-time curve and is therefore not only the consequence of the increased $K_{d}$. To demonstrate this, we overlaid the simulations with simulations of hypothetical compounds that have the same $K_{\mathrm{d}}$ values as aclidinium and PF-3635659, but the $k_{\text {off }}$ value of ipratropium. These hypothetical compounds only showed a rightward shift of the occupancy-time curve and had considerably shorter duration of target occupancy compared with aclidinium and PF-3635659, respectively.

Another example of a decrease in $k_{\text {off }}$ that leads only to an affinity-driven prolongation of target occupancy is given in the initial opinion article of Copeland and colleagues ${ }^{1}$, in which the increasing duration of target occupancy was (incorrectly) used to demonstrate the influence of drug-target residence time. In these simulations, the $k_{\text {off }}$ values are all much higher than the elimination rate constant and the decrease in $k_{\text {off }}$ only results in a rightward shift of the occupancy-time curve, as in FIG. 1 b.

The fact that a higher drug concentration or increased affinity leads to an increased duration of drug effects has been described in quantitative terms since the early days of pharmacokinetic/pharmacodynamic (PK/PD) modelling ${ }^{7}$. More recently, the relationship between target saturation and the duration of target occupancy has also been discussed more quantitatively with respect to drugtarget binding kinetics; for example, see $\mathrm{REFS}^{4,8}$. In a previous publication ${ }^{4}$, we investigated with mathematical approximations when drug-target dissociation (that is, $k_{\text {off }}$ ) becomes the rate-limiting step for the duration of drug action compared with pharmacokinetics, target saturation and rebinding (that is, the influence of target binding on the drug concentration around the target). Although the influence of target saturation on the duration of target occupancy is mathematically well defined, the relevance of target saturation for the influence of $k_{\text {off }}$ on the duration of target occupancy has not been a focus of previous articles and has been ignored in several papers focusing on the influence of $k_{\text {off }}$ on target occupancy.

To find the $k_{\text {off }}$ value that gives a significant prolongation of target occupancy, we identified previously for what values of target occupancy the elimination rate constant $\left(k_{\mathrm{el}}\right)$ of the drug from plasma would have less influence on the duration of target occupancy than $k_{\text {off }}^{4}$ (that is, for what values the $k_{\text {off }}$ is the main determinant of the duration of target occupancy). We performed this approximation by assuming that the slowest step on the path of target dissociation and free drug elimination determines the decline rate of target occupancy. To do this analysis correctly, target saturation needs to be taken into account. The influence of target saturation becomes 
a
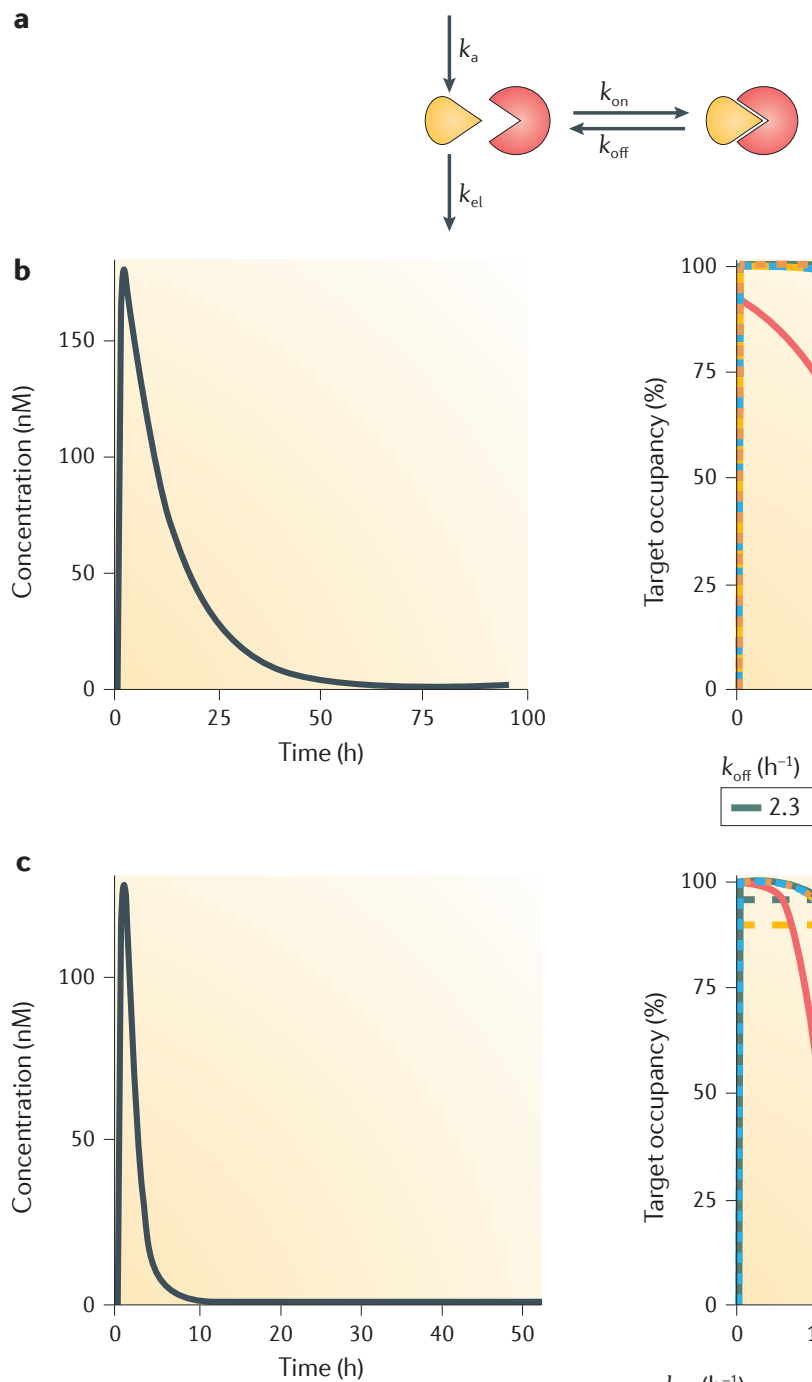
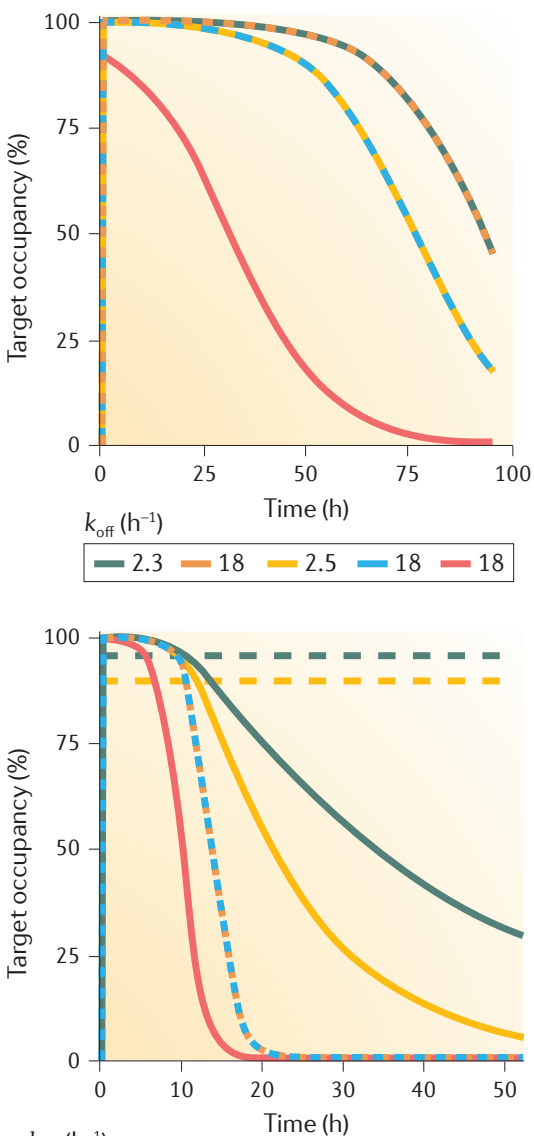

$k_{\text {off }}\left(\mathrm{h}^{-1}\right)$

$-0.029-0.072-4.0-4.0-4.0$

Figure 1 | Simulation of the implications of high target occupancy for drug-target residence time. a | Model used in the simulations. Here, $k_{\mathrm{a}}$ and $k_{\mathrm{el}}$ represent the first-order absorption constant $\left(3.0 \mathrm{~h}^{-1}\right)$ and elimination rate constant, respectively, while $k_{\text {on }}$ and $k_{\text {off }}$ represent the second-order association rate constant and the first-order dissociation rate constant, respectively. $\mathbf{b}$ | Simulations of plasma drug concentrations (left panel) and the resulting target occupancy profiles (right panel) for different compounds. The solid lines represent the HIV protease inhibitors amprenavir (red), atazanavir (yellow) and lopinavir (green), with $k_{\text {on }}$ values of $1.1,6.2$ and $23 \mathrm{nM}^{-1} \mathrm{~h}^{-1}$, respectively. The dotted lines represent hypothetical compounds with the same $K_{d}$ values as atazanavir and lopinavir but the same $k_{\text {off }}$ value as amprenavir, leading to $k_{\text {on }}$ values of 44 (blue) and 176 (orange) $\mathrm{nM}^{-1} \mathrm{~h}^{-1}$, respectively. The target concentration was set at $1 \mathrm{pM}$. The elimination rate constant was $0.082 \mathrm{~h}^{-1}$. The dose in the absorption compartment corresponds to an initial plasma concentration of $200 \mathrm{nM}$, if absorption would be immediate. All plasma concentration profiles overlap. For the associated differential equations, see Supplementary information S1, and for the R simulation script, see Supplementary information S2. Similar simulations can be performed online (see Related links). c|Simulations of plasma drug concentrations (left panel) and the resulting target occupancy profiles (right panel) for different compounds. The solid lines represent the muscarinic receptor antagonists ipratropium (red), aclidinium (yellow) and PF-3635659 (green), with $k_{\text {on }}$ values of $15,4.0$ and $1.4 \mathrm{nM}^{-1} \mathrm{~h}^{-1}$, respectively. The dotted lines represent hypothetical compounds with the same $K_{d}$ values as aclidinium and PF-3635659 but the same $k_{\text {off }}$ value as ipratropium, leading to $k_{\text {on }}$ values of 198 (blue) and 218 (orange) $\mathrm{nM}^{-1} \mathrm{~h}^{-1}$, respectively. All plasma concentration profiles overlap. The concentration of the target (the muscarinic $\mathrm{M}_{3}$ receptor was set at $1 \mathrm{pM}$. The elimination rate constant was $0.69 \mathrm{~h}^{-1}$. The dose in the absorption compartment corresponds to an initial plasma concentration of $200 \mathrm{nM}$, if absorption is immediate. The dashed horizontal lines denote the situation in which the target fraction bound equals $1-k_{\text {off }} / k_{\text {el }}$ (see text). Below that line, the condition is met for which $k_{\text {off }}$ is the main determinant of the decline rate of target occupancy.

most significant for target occupancies $>50 \%$, where a $1 \%$ increase in drug concentration leads to $<0.5 \%$ increase in occupancy, while at target occupancies approaching $0 \%$, a $1 \%$ increase in drug concentration leads to a $1 \%$ increase in target occupancy. The horizontal lines in FIG. 1c illustrate that slow drug-target dissociation is the main determinant of the duration of target occupancy if both the dissociation rate constant and the target occupancy have values such that: $B F<1-k_{\text {off }} / k_{\text {el }}$ (in which $B F$ is the target fraction bound). It should be noted that this equation is rewritten from an approximation of the simple drugtarget binding model and only holds for this model if the target concentration is lower than the ratio $k_{\mathrm{el}} / k_{\mathrm{on}}$, as described previously $\mathrm{y}^{4}$. Of note, the target occupancy-time curves in FIG. 1 b,c are independent of this approximation, as they are simulated with the original equations, not with the approximations. Only the horizontal lines in FIG. 1c are based on the approximation.

From this equation, it follows that when the clinical situation requires a high target occupancy (as can be expected especially for antagonists for chronic diseases with daily or less frequent dosing), then $k_{\text {off }}$ will need to be much smaller than $k_{\text {el }}$ for it to become the main determinant of the duration of target occupancy.

These findings can be applied directly to the selection of drug candidates. An example in which our insights could have been applied is the study of Lindström and colleagues ${ }^{9}$, which compared the in vivo drug effects of three neurokinin $1\left(\mathrm{NK}_{1}\right)$ receptor antagonists with their pharmacokinetics. Aprepitant demonstrated a much longer duration of drug effect, which can clearly be attributed to target saturation, considering that the effect is close to $100 \%$ for a long time in the experiment, followed by a steep decline. In contrast, the authors conclude that the duration of the effect of aprepitant cannot be explained by its pharmacokinetics. The other two compounds in this study did not show this target saturation and the authors conclude that this is probably explained by their faster binding kinetics. However, our findings above indicate that the increased duration of the aprepitant effect is mainly due to its high brain concentrations compared with its affinity, which causes target saturation.

Our quantitative approximations can also be applied to the decision as to whether to consider drug-target residence time in hit and/or lead selection. For C-C chemokine receptor type 2 (CCR2) antagonists, an occupancy of $>90 \%$ is considered to be required for a sufficient drug effect. Based on the 
equation above, this means that the dissociation half-life needs to be 10 times larger than the plasma half-life in order for it to be the main determinant of target occupancy. As the plasma half-life of the CCR2 antagonist identified by Bot and colleagues was 11 hours ${ }^{10}$, this means that the dissociation half-life would need to be 110 hours or longer before it became the main determinant of the duration of drug effect. In combination with the knowledge that such long dissociation halflives are rarely observed ${ }^{4}$, this suggests that seeking to prolong the dissociation half-life should not be prioritized when searching for CCR2 antagonists with a prolonged duration of effect, or for other drug targets for which high occupancies are considered essential to achieve the desired pharmacological effect.

\section{Conclusion}

Target saturation is an important factor that should be included in the analysis of the influence of drug-target binding kinetics on target occupancy. By doing so, drug discovery scientists would be better equipped to decide on the relevance of drug-target binding kinetics for each specific project, depending on the required level of target occupancy and the (predicted) pharmacokinetics.

Wilhelmus E. A. de Witte ${ }^{*}$, Meindert Danhof ${ }^{\prime}$, Piet H. van der Graaf ${ }^{1,2}$ and Elizabeth C. M. de Lange

${ }^{1}$ Division of Pharmacology, Leiden Academic Centre for Drug Research, Leiden University, Leiden, The Netherlands.

${ }^{2}$ Certara Quantitative Systems Pharmacology, Canterbury Innovation Centre, Canterbury, UK.

*e-mail: wilbertdew@gmail.com

doi: $10.1038 /$ nrd.2018.234

Published online 28 Dec 2018

1. Copeland, R. A., Pompliano, D. L. \& Meek, T. D. Drug-target residence time and its implications for lead optimization. Nat. Rev. Drug Discov. 5,730-739 (2006).

2 Tonge, P. J. Drug-target kinetics in drug discovery. ACS Chem. Neurosci. 9, 29-39 (2018).

3. Folmer, R. H. A. Drug target residence time: a misleading concept. Drug Discov. Today $23,12-16$ (2018).

4 de Witte, W. E. A., Danhof, M., van der Graaf, P. H. \& de Lange, E. C. M. In vivo target residence time and kinetic selectivity: the association rate constant as determinant. Trends Pharmacol. Sci. 37, 831-842 (2016).

5. Dahl, G. \& Akerud, T. Pharmacokinetics and the drug -target residence time concept. Drug Discov. Today 18, 697-707 (2013).
6 Keserü, G. M. \& Swinney, D. C. Thermodynamics and Kinetics of Drug Binding (Wiley-VCH Verlag $\mathrm{GmbH} \&$ Co. KGaA, Heidelberg, Germany, 2015).

7. Levy, G. Kinetics of pharmacologic effects. Clin. Pharmacol. Ther. 7, 362-372 (1966).

8. Vauquelin, G. \& Van Liefde, I. Slow antagonist dissociation and long-lasting in vivo receptor protection. Trends Pharmacol. Sci. 27, 356-359 (2006).

9. Lindström, E. et al. Neurokinin 1 receptor antagonists: correlation between in vitro receptor interaction and in vivo efficacy. J. Pharmacol. Exp. Ther. 322 1286-93 (2007).

10. Bot, I. et al. A novel CCR2 antagonist inhibits atherogenesis in apoE deficient mice by achieving high receptor occupancy. Sci. Rep. 7, 52 (2017).

\section{Acknowledgements}

The authors are part of the K4DD consortium, which is supported by the Innovative Medicines Initiative Joint Undertaking (IMI JU) under grant agreement no 115366 . The IMI $\mathrm{JU}$ is a project supported by the EU's Seventh Framework Programme (FP7/2007-2013) and the European Federation of Pharmaceutical Industries and Associations (EFPIA).

Competing interests

The authors declare no competing interests.

Supplementary information

Supplementary information is available at https://doi.org/ 10.1038/nrd.2018.234

\section{RELATED LINKS}

Absorption, binding and elimination model: https://

wilbertdewitte.shinyapps.io/absorption binding elimination

ALL LINKS ARE ACTIVE IN THE ONLINE PDF 